\title{
Coopérer dans une équipe de projet entrepreneurial: une affaire de leadership ?
}

\author{
CAROLINE VERZAT \\ ESCP Europe, Paris, cverzat@escp.eu \\ NOREEN O'SHEA \\ ESCP Europe, Paris, noshea@ escp.eu
}

\begin{abstract}
Résumé
L'apprentissage coopératif, qualifié par le partage et l'entraide entre les apprenants, représente un des 4 piliers de référence pour la pédagogie par projets dans le domaine de l'entrepreneuriat (Surlemont et Kearney, 2009). Mais, à ce jour, il n'existe pas de description fine des différents niveaux de coopération générés par les équipes-projet ni d'étude de l'interdépendance effective entre coopération et réussite des projets. Notre recherche tente d'y répondre. Nous avons choisi une approche qualitative basée sur l'analyse des récits d'expérience vécue par 13 équipesprojets en entrepreneuriat, soit 66 étudiants inscrits en Master 1 ou 2 dans deux écoles de commerce en France. Les résultats des analyses, obtenus à l'aide du logiciel NVivo ont permis d'identifier 4 idéaux-types d'équipes. Dans deux types d'équipes, on peut distinguer deux natures différentes de pratiques coopératives ; un troisième s'appuie plutôt sur des pratiques collaboratives et le quatrième n'est ni coopératif, ni collaboratif. Les différents types de coopération et de collaboration ont également une relation directe avec la configuration de leadership établie, le niveau d'implication des membres et la qualité de la production.
\end{abstract}

\begin{abstract}
Cooperative learning, characterised by the enabling of mutual support among learners, is one of the 4 reference pillars underlying pedagogy in project-based learning in the field of entrepreneurship (Surlemont and Kearney, 2009). However, to date, literature does not offer any clear description of the different levels of cooperation generated amongst teams, nor does it explain if and how it influences their successful outcomes. This is the purpose of the present study. We adopted a qualitative approach, based on the analysis of the written narratives of 66 Master students enrolled in 2 French business schools, all involved in developing a real-life entrepreneurial project. The results of the analysis, obtained with NVivo software, enabled us
\end{abstract}


to identify 4 ideal types of teams, which highlight different degrees and types of cooperation. There was a direct link to the configuration of leadership adopted, the level of implication displayed by the members and the quality of the production outcomes.

\section{Mots-clés}

Coopération, collaboration, leadership, éducation entrepreneuriale.

\section{Key words}

Cooperation, collaboration, leadership, entrepreneurship education.

\section{Introduction}

La pédagogie par projets est courante dans le domaine de l'entrepreneuriat afin d'apprendre la démarche de création d'entreprise par l'expérience. Parmi les quatre piliers pédagogiques de référence dans la discipline ${ }^{1}$, Surlemont et Kearney (2009) préconisent la coopération au sens d'un partage et d'une entraide entre les apprenants. Elle est supposée offrir de meilleures occasions de mise en pratique, favoriser les contacts et la proximité entre les apprenants ainsi qu'avec les partenaires extérieurs, renforcer la motivation des apprenants, et finalement maximiser les apprentissages. De même Pelletier (2005, p. 18) prescrit une culture coopérative pour soutenir les projets entrepreneuriaux : «Si des élèves se retrouvent ensemble pour réaliser un projet collectif, ils auront à prendre des initiatives, à proposer des buts et des moyens, à s'engager solidairement dans des tâches complémentaires; ils devront coordonner leurs activités, travailler en équipe, s'encourager, échanger de l'information, gérer les contraintes, consentir à faire des efforts pour respecter les échéances. Chacun étant une ressource pour luimême et pour les autres, devra se mettre à l'épreuve dans une situation globale inédite. Il s'ensuit une responsabilité solidaire, une part de devoir qu'impose, dans les circonstances, l'urgence de faire ce qu'il faut, enfin la réussite partagée. Après cela, on se sentira plus estimable et on fera un retour sur l'histoire du projet et du groupe, sur les points forts, sur les points faibles qu'on pourrait améliorer, et surtout, on voudra recommencer. »

Bien que ces recommandations aient été formulées pour l'enseignement primaire et secondaire, ces auteurs sont constamment cités par des enseignants en entrepreneuriat dans l'enseignement

\footnotetext{
${ }^{1}$ Les quatre principes recensés par Surlemont et Kearney sont l'apprentissage responsabilisant, l'apprentissage « en direct », l'apprentissage coopératif et l'apprentissage réflexif.
} 
supérieur. Or la philosophie coopérative ne correspond pas forcément avec la réalité vécue par les étudiants en entrepreneuriat. Nos enquêtes antérieures sur des équipes de niveau master mettent en évidence des perceptions d'efficacité groupale variables selon les groupes (Verzat, O'Shea, et Jore 2017) laissant suspecter des niveaux de coopération différents. Cette intuition est confirmée par une étude qualitative fine sur un cas montrant que les étudiants peuvent atteindre une haute performance en termes de réussite du projet sans pour autant prendre tous des initiatives, s'entraider ou se montrer solidaires (Foliard et Le Pontois, 2017). Mais il n'existe pas à notre connaissance de littérature concernant les critères qui permettraient de décrire les différents niveaux de coopération des équipes et d'apprécier leur influence sur la réussite des projets. Nous proposons de combler ce manque grâce à l'identification d'idéauxtypes d'équipe caractérisant à la fois les niveaux de coopération établis au sein du groupe et le niveau de réussite de leur projet entrepreneurial.

Notre recherche s'appuie sur l'analyse du leadership utilisée dans le cas d'équipes d'étudiants ingénieurs en Apprentissage par Problème et par Projet (APP) (Verzat, O’Shea, et Raucent, 2015). Cette recherche avait mis en évidence l'influence des configurations de leadership sur les résultats des équipes projet en termes de production, satisfaction et apprentissage. Nous avions notamment montré qu'il existait différentes modalités de répartition des tâches, d'allocation des responsabilités relevant soit de leaders reconnus au sein du groupe, soit de partage de fonctions de leadership entre les différents membres. Ces modalités se traduisaient par des niveaux d'implication variables laissant suspecter l'existence de plusieurs degrés possibles de collaboration ou de coopération au sein du groupe. Notre article se propose donc de qualifier la relation entre les différentes configurations de leadership établies et les niveaux de collaboration ou de coopération effectifs, puis d'en apprécier l'influence sur la réussite des projets telle qu'elle est perçue par les membres des groupes et par les parties prenantes extérieures.

Les données analysées ici sont les récits de projets écrits a posteriori par 66 étudiants engagés dans 13 équipes entrepreneuriales dans un but d'analyse réflexive de leurs apprentissages. Après avoir présenté notre cadre théorique et la problématique qui en découle puis la méthodologie d'analyse qualitative utilisée, nous présenterons et discuterons les résultats de cette recherche. 


\section{Cadre théorique et problématique}

Il n'est pas aisé de définir la coopération car elle est souvent confondue avec la collaboration, les notions étant voisines. En nous appuyant sur les synthèses de Baudrit (2007), PeyratMalaterre (2011), Go (2013) et Davidson et Major (2014), nous retenons que les deux approches partagent la visée de faire travailler les groupes dans un but commun en s'appuyant sur l'implication de tous, la responsabilité de chacun et l'interdépendance entre les membres. Mais l'interdépendance ne porte pas sur les mêmes objets, n'y est pas régulée de la même manière par les enseignants et ne correspond pas exactement aux mêmes valeurs et finalités. L'approche coopérative structure l'interdépendance au niveau des objectifs, des tâches, des ressources, des rôles et des récompenses, tandis que l'approche collaborative porte seulement sur les objectifs, les tâches et les ressources. L'approche coopérative, plus généralement pratiquée au primaire, donne à l'enseignant un rôle central en vue de travailler les habiletés coopératives. L'enseignant structure la tâche, clarifie les rôles et responsabilités requises pour l'accomplir, et explicite la nature des interactions et des échanges nécessaires. Il a aussi un rôle de facilitation au sein des groupes afin d'entraîner les compétences sociales des apprenants. En revanche, dans l'approche collaborative, plus généralement pratiquée dans l'enseignement secondaire et dans le supérieur, l'enseignant laisse les apprenants libres de s'auto-organiser, lui-même se positionnant comme une personne ressource sans rentrer dans le contrôle interne opéré par le groupe. Ce qui peut conduire à interpréter l'approche coopérative comme un préalable à l'apprentissage collaboratif. Enfin, les deux approches mettent l'accent sur des finalités et valeurs distinctes : «Si dans la collaboration, c'est la production qui compte, la coopération enveloppe l'effectuation de la tâche d'une préoccupation de l'art de vivre ensemble, dont la valeur prend parfois le pas sur la production elle-même »(Go, 2013, p. 52). Les deux approches supposent équité et réciprocité de la part des apprenants, mais la collaboration valorise plus la solidarité, tandis que la coopération valorise davantage la générosité et le partage.

La coopération dans un sens général (implication de tous et interdépendance entre les membres d'un groupe) est souvent prescrite chez les étudiants du supérieur afin de favoriser l'accomplissement de buts communs dans le travail en équipe. Mais étant laissée à la responsabilité des groupes (approche collaborative) ou régulée de manière différente selon les enseignants (approche coopérative), ses niveaux d'accomplissement effectifs ne sont pas connus avec précision. Pourtant, des limites sont identifiées dans la littérature. Dans l'apprentissage collaboratif, supposant une égalité absolue de statut «les élèves ne se 
responsabilisent peut-être pas de façon équivalente en leur sein, leurs niveaux d'implication ne sont certainement pas similaires » (Baudrit, 2007, p. 125). Dans l'apprentissage coopératif, l'hétérogénéité recherchée en termes de genres, d'origine ou de niveau de connaissances afin de favoriser les conflits socio-cognitifs «oriente vers des positions sociales distinctes préjudiciables » (Baudrit, 2007, p. 126). L'un des points particulièrement délicats est le phénomène des passagers clandestins, qui laissent les autres membres du groupe prendre la plus grande part du travail sans s'y impliquer au même degré (Slavin, 1995). Le deuxième est la distribution de l'autorité au sein du groupe : "Des élèves peuvent adopter des rôles de "leaders" ou de "suiveurs" (Baudrit, 2007, p. 124). C'est à cette dernière dimension que nous allons nous attarder, nous verrons qu'elle peut avoir des liens avec le problème des passagers clandestins.

La notion de leadership est considérée en psychologie sociale comme la variable centrale permettant de comprendre l'allocation des tâches, des responsabilités et de l'autorité déterminant la performance des équipes. Elle est souvent associée à la figure d'un leader central dirigeant le groupe et influençant des suiveurs. Mais quelques auteurs ont montré que le leadership peut également être réparti entre les différents membres du groupe, en particulier dans les équipes non hiérarchiques. Le leadership est alors conceptualisé comme une « collection de rôles et de comportements qui peuvent être distribués, partagés, inter changés séquentiellement ou simultanément » (Barry, 1991, p. 34). Quatre fonctions sont identifiées dans la littérature, deux principales à assurer tout au long du projet : organisateur (coordonner le travail), social (prendre soin des besoins d'harmonie du groupe, gérer les conflits), et deux fonctions ponctuelles : visionnaire (initier une vision commune), et ambassadeur (gérer les relations avec l'extérieur).

En s'appuyant sur ces concepts, notre précédente recherche sur les équipes d'APP à l'École Polytechnique de Louvain (Verzat, O'Shea, et Raucent, 2015) a mis en évidence quatre configurations de leadership impactant la production, la satisfaction et l'apprentissage des étudiants : 1) le leadership distribué entre plusieurs étudiants endossant des fonctions de leadership variées, ce qui aboutit à une production, un niveau d'apprentissage et une satisfaction supérieurs à celle des autres configurations, 2) le leadership focalisé sur un leader organisateur unique conduisant à un bon niveau de production mais une satisfaction et un apprentissage variables selon les membres, 3) l'illusion de leadership lorsque le groupe est scindé entre des producteurs et des passagers clandestins, générant une satisfaction réduite et une production limitée, 4) l'absence de leadership où peu de choses s'apprennent et se produisent. Toutefois, la manière dont les différentes fonctions sont prises en charge entre les membres n'avait pas été 
étudiée avec précision dans cette recherche. De plus, nous avions également noté qu'une fonction d'expert-pédagogue non identifiée dans la littérature sur le leadership distribué, pourrait davantage être investiguée. Elle permettrait de rendre compte de l'influence de l'un ou plusieurs membres du groupe, reconnus par les autres comme experts sur un domaine et prêts à partager leurs connaissances avec eux afin d'améliorer la compréhension de chacun au service du projet.

À la suite de cette revue de littérature, nous présumons que l'analyse des configurations de leadership pourrait aider à clarifier les niveaux de collaboration/coopération repérables au sein des équipes d'étudiants en entrepreneuriat. Le but de notre communication est donc de clarifier les configurations de leadership effectives dans les différentes équipes et d'étudier leur relation avec les niveaux de collaboration/coopération perçues par les étudiants ainsi que leur impact sur la qualité de la production atteinte.

\section{Méthodologie}

Nous avons privilégié une approche qualitative basée sur des récits d'expérience entrepreneuriale vécue par les étudiants. Notre échantillon est composé de 13 équipes de projet, soit 66 étudiants inscrits en Master 1 ou 2 dans 2 écoles de commerce (5 équipes dans une école européenne, 8 équipes dans une école française). Dans les deux écoles, les étudiants ont réalisé un projet entrepreneurial en 3 mois (école européenne) et 5 mois (école française). Dans les deux contextes, le projet se termine par la rédaction d'un business plan ainsi qu'une présentation orale suivie de questions/réponses par un jury extérieur identique pour toutes les équipes. Le jury est composé de plusieurs professionnels extérieurs à titre d'investisseurs ou membres de réseaux d'accompagnement en entrepreneuriat. Après la présentation finale, chaque étudiant devait faire un retour d'expérience à travers un récit écrit répondant à quatre thématiques ${ }^{2}$. Les récits comportent en moyenne 1650 mots par étudiant (3 à 10 pages).

\footnotetext{
${ }^{2}$ Les quatre thématiques sont les suivantes : 1) Raconter les faits. 2) Analyser les ressentis associés à ces faits. 3) Tirer les leçons. 4) Prévoir l'action future. Les récits individuels représentaient $40 \%$ de la note du cours. Les $60 \%$ restants étaient attribués collectivement (présentation orale + dossiers de business plan). Une grille critériée de notation du récit individuel était fournie aux étudiants en amont. Les points sont attribués selon que les critères sont non vérifiés ( 0 point $)$, partiellement vérifiés (1 point) ou totalement vérifiés ( 2 points). Les critères sont les suivants : faits précis, faits significatifs, clarification de l'action de chaque membre, ressentis repérés chronologiquement, ressentis analysés pour soi, sensibilité aux ressentis des autres membres, nombre de leçons tirées $>=3$, leçons des réussites comme des échecs, repérage des ressources les plus aidantes, nombre de résolutions prises $>=3$, lisibilité du récit, longueur $>1000$ mots.
} 
Pour analyser les récits, les deux chercheures se sont appuyées sur le logiciel NVivo pour réaliser un codage thématique en confrontant systématiquement leurs interprétations afin de parvenir à un accord de $100 \%$. Quatre catégories de codage ont été construites à partir de nos recherches antérieures et affinées à la lecture des récits : la coopération perçue, le type de leadership mis en œuvre, le degré d'implication des membres au cours du projet, le niveau de qualité de la production, appréciée par les membres de l'équipe et par le jury extérieur. Nous précisons le contenu de ces thématiques ci-après.

En ce qui concerne le niveau de coopération perçue, l'analyse a permis de caractériser le degré de coopération, la nature de la coopération, ainsi que l'existence de conflits résolus ou non ${ }^{3}$. À propos de l'exercice du leadership, deux indicateurs ont été repérés dans les récits. Le premier concerne la reconnaissance du leadership par les membres du groupe, signalée par l'utilisation des mots leader, leadership, manager ou chef à propos d'un membre du groupe. Le deuxième indicateur est l'explication fournie par le narrateur du récit d'expérience sur la manière dont lui-même ou les autres membres du groupe suivent cette personne. Lorsque l'une ou l'autre de ces explications étaient présentes, nous avons également cherché à analyser la fonction du leadership décrite à propos du leader en question: visionnaire, ambassadeur, social, organisateur et/ou expert pédagogue. Les niveaux d'implication des membres ont été repérés à partir de tous les commentaires portant sur l'implication en général et l'égalité (ou non) des contributions des membres. Enfin le niveau de qualité de la production a été apprécié à partir des commentaires des étudiants à propos de leurs résultats ainsi qu'à partir de ce qu'ils rapportaient des réactions des membres du jury à l'issue des présentations. La perception externe de la qualité a également été appréciée à partir des notes attribuées par les jurys dans chaque école, donnant lieu à un classement au sein de la classe.

À partir des valeurs de codage sur chaque thème (nombre de verbatim moyen par étudiant de l'équipe ${ }^{4}$ ), nous avons comparé les équipes entre elles afin d'identifier des idéaux-types de coopération, de leadership, et de réussite perçus.

\footnotetext{
${ }^{3}$ Trois catégories de verbatim concernant les conflits ont émergé : ceux qui se terminent bien (constructifs), ceux qui se terminent mal (destructeurs) et ceux que les étudiants perçoivent a posteriori mais sont restés latents faute d'avoir été exprimés et gérés (non exprimés). Pour simplifier la lecture dans le texte, nous mentionnons le nombre de verbatim sur les différentes natures de conflits par catégorie d'équipe, mais l'analyse de départ pour aboutir aux idéaux-types a porté sur le nombre moyen de verbatim par étudiant par équipe.

${ }^{4}$ Le nombre de verbatim codés par équipe a été ensuite divisé par le nombre d'étudiants afin de tenir compte de la taille variable des équipes (4 à 7 étudiants). Sur chaque dimension, le chiffre pris en compte pour l'analyse est compris entre 0 ( $\mathrm{si}$ la dimension est totalement absente car aucun étudiant de l'équipe ne l'a exprimée) et 3,2 (valeur maximale obtenue correspondant au fait que cette dimension a été citée 3,2 fois en moyenne par étudiant de l'équipe). Les dimensions les plus citées sont le niveau de coopération ( $\max =3,2)$, le niveau d'implication
} 


\section{Résultats}

Nous avons identifié quatre idéaux-types d'équipes qui mettent en évidence différents degrés et types de coopération en relation directe avec la configuration de leadership établie, le niveau d'implication des membres et la qualité de la production. Le tableau 1 ci-dessous résume les résultats obtenus.

Tableau 1 - Configuration de coopération et de leadership dans les équipes

\begin{tabular}{|c|c|c|c|c|c|}
\hline & & $\begin{array}{l}\text { Groupe à leadership } \\
\text { distribué ( } 3 \text { équipes) }\end{array}$ & $\begin{array}{l}\text { Groupe cohésif } \\
\text { (3 équipes) }\end{array}$ & $\begin{array}{l}\text { Groupe à leader } \\
\text { organisateur } \\
\text { (2 équipes) }\end{array}$ & $\begin{array}{l}\text { Groupe à leadership } \\
\text { en échec } \\
\text { (5 équipes) }\end{array}$ \\
\hline \multirow{3}{*}{$\begin{array}{l}\text { Coopération } \\
\text { perçue }\end{array}$} & Niveau de coopération & Fort & Moyen ou fort & Moyen ou faible & Faible \\
\hline & $\begin{array}{l}\text { Nature de la } \\
\text { coopération }\end{array}$ & $\begin{array}{l}\text { Solidarité, entraide et } \\
\text { reconnaissance des } \\
\text { autres afin de relever } \\
\text { les défis }\end{array}$ & $\begin{array}{l}\text { Cohésion recherchée } \\
\text { au niveau du groupe : } \\
\text { complémentarité et } \\
\text { bonne entente }\end{array}$ & $\begin{array}{l}\text { Collaboration par } \\
\text { délégation de tâches }\end{array}$ & $\begin{array}{l}\text { Manquante, } \\
\text { discours de façade } \\
\text { au mieux }\end{array}$ \\
\hline & Conflits & $\begin{array}{l}\text { Constructifs grâce à des } \\
\text { efforts de médiation }\end{array}$ & Rares & $\begin{array}{l}\text { Destructeurs ou } \\
\text { latents }\end{array}$ & $\begin{array}{l}\text { Destructeurs ou } \\
\text { latents }\end{array}$ \\
\hline \multirow[t]{2}{*}{ Leadership } & $\begin{array}{l}\text { Reconnaissance du } \\
\text { leadership par les } \\
\text { membres du groupe }\end{array}$ & Forte & Faible & Forte & Absente \\
\hline & $\begin{array}{l}\text { Fonctions de } \\
\text { leadership exercées } \\
\text { par ordre } \\
\text { d'importance }\end{array}$ & $\begin{array}{l}\text { 1- social } \\
2 \text { - organisateur } \\
3 \text { - expert pédagogue } \\
4 \text { - visionnaire } \\
5 \text { - ambassadeur }\end{array}$ & $\begin{array}{l}1 \text { - expert pédagogue } \\
2 \text { - ambassadeur } \\
3 \text { - social } \\
4 \text { - organisateur }\end{array}$ & 1 - organisateur & $\begin{array}{l}\text { 1- organisateur } \\
\text { (en échec) }\end{array}$ \\
\hline $\begin{array}{l}\text { Implication } \\
\text { des membres }\end{array}$ & $\begin{array}{l}\text { Niveau d'implication } \\
\text { des différents } \\
\text { membres du groupe }\end{array}$ & $\begin{array}{l}\text { Inégal mais se renforce } \\
\text { au fur et à mesure }\end{array}$ & $\begin{array}{l}\text { Moyen ou fort de tous } \\
\text { les membres }\end{array}$ & $\begin{array}{l}\text { Inégal tout au long } \\
\text { du projet (passagers } \\
\text { clandestins) }\end{array}$ & $\begin{array}{l}\text { Désengagement } \\
\text { progressif de tous }\end{array}$ \\
\hline \multirow[t]{2}{*}{ Production } & $\begin{array}{l}\text { Qualité perçue par les } \\
\text { membres du groupe }\end{array}$ & Supérieure aux attentes & Conforme aux attentes & $\begin{array}{l}\text { Conforme aux } \\
\text { attentes ou } \\
\text { inférieure }\end{array}$ & $\begin{array}{l}\text { Inférieure aux } \\
\text { attentes }\end{array}$ \\
\hline & $\begin{array}{l}\text { Qualité attestée par le } \\
\text { jury }\end{array}$ & Supérieure & $\begin{array}{l}\text { Variable } \\
\text { ( } 2 \text { supérieures, } 1 \\
\text { inférieure) }\end{array}$ & Moyenne & Inférieure \\
\hline
\end{tabular}

Les récits des équipes à leadership distribué sont caractérisés par trois éléments : l'utilisation du terme de leadership ou leader à propos de plusieurs fonctions de leadership et de plusieurs membres du groupe, une forte perception de coopération malgré l'existence de nombreux conflits et enfin un niveau de qualité de production élevé. Le leadership est spontanément

$(\max =2,45)$, le leadership exprimé $(\max =2,43)$, la fonction de leader organisateur $(\max =2,83)$, la fonction de leader social $(\max =1,83)$, la fonction de leader ambassadeur $(\max =1,67)$, la fonction de leader expert-pédagogue $(\max =1,60)$, la qualité de production reconnue par les membres du groupe $(\max =2,40)$. 
reconnu par au moins la moitié des membres des équipes ${ }^{5}$. L'histoire des trois équipes montre que des leaders ont émergé naturellement mais que la distribution des fonctions a évolué au fur et à mesure des besoins des personnes et du projet ${ }^{6}$ grâce à une gestion consciente s'appuyant sur les catégories de leadership présentées en cours ${ }^{7}$. «J'ai appris à partager le leadership, ce que je n'avais pas fait jusqu'à présent dans mes études. [ ] Chacun peut être à son tour le leader visionnaire, organisateur, facilitateur, ambassadeur ou le knowledge manager. » Nous avons observé la présence des cinq fonctions dans 2 des trois équipes concernées (trois fonctions dans la $3^{\mathrm{e}}$ ) avec une prééminence de la fonction sociale qui permet de rendre les conflits constructifs : «Elle a su manager les qualités de tout le monde, en maintenant un climat de bonne ambiance dans le groupe malgré les désaccords. » «J'ai donc pris à part A. et M. et je leur ai expliqué que s'il y avait un problème, ils devaient en parler et le régler. Tout passe par la communication, il fallait qu'on s'entraide. On est avant tout une équipe. » La coopération est perçue comme forte. Les étudiants soulignent tous l'entraide et la solidarité qui permet d'affronter ensemble la charge de travail, les retours défavorables ou encore les déficits d'implication (1,2 à 3,2 verbatim par étudiant dans les 3 équipes concernées). «Tout au long du projet, nous sommes restés soudés malgré les difficultés et les déceptions. » « Heureusement que l'entraide est présente au sein de cette équipe et que mes camarades ont pris en charge une partie de mon travail sur les autres matières pour me laisser le temps d'animer les réseaux. » «En observant les autres groupes, notamment lorsque les tensions se faisaient sentir, lorsque certains en font moins que d'autres par exemple, je me suis aperçue que nous étions tous très impliqués dans ce que nous entreprenions et que l'entraide était la clé pour réussir ces projets. » Les conflits sont nombreux (26 verbatim sur un total de 87 dans l'ensemble du corpus) mais la majorité d'entre eux concernent des conflits gérés et résolus (15 verbatim sur 26) : «Il y a eu des incompréhensions, certains étaient sous tension à cause du degré

\footnotetext{
${ }^{5}$ Les 3 équipes concernées (sur 13) concentrent 12 verbatim sur les 48 de notre corpus qui attestent de la reconnaissance explicite d'un leader. Dans chacune de ces équipes, il existe au moins 3 formes de leadership exercées (valeurs comprises entre 0,5 et 1,83 selon les fonctions exercées).

${ }^{6}$ Dans les trois équipes, le développement des fonctions de leadership suit plusieurs processus d'évolution : appui au départ sur un leader naturel organisateur, lequel prend progressivement conscience du besoin de partage des autres équipiers et s'appuie sur un co-leader social notamment pour résoudre les conflits et rejoindre les membres les plus éloignés du projet, ce qui le soulage et l'apaise ( 2 cas) ou bien présence dès le départ de personnalités fortes qui se partagent le leadership visionnaire et organisateur $(1$ cas). Puis dans les 3 cas, l'émergence de nouveaux besoins du projet fait émerger de nouvelles fonctions : ambassadeur pour traiter les relations avec l'extérieur ( 2 cas), expert-pédagogue afin de donner de l'aide sur des parties techniques, centraliser et valider la cohérence des données du projet au sein des documents partagés ( 2 cas).

${ }^{7}$ Nous n'avons relevé dans aucune autre catégorie d'équipe des références au vocabulaire sur la distribution des fonctions de leadership expliquées en cours.
} 
d'implication des autres membres. Nous avons donc choisi de communiquer pour résoudre le conflit et nous avons pu repartir sur de bonnes bases jusqu'à l'aboutissement du projet final. » «Même si les désaccords menaient parfois à des engueulades, nous essayons d'en tirer le maximum de leçons à chaque fois que l'orage passait. » Ce haut niveau de coopération produit une «énergie, une communion, une émulation, une adrénaline particulière ». La qualité de la production résultante étonne les membres de l'équipe eux-mêmes. Elle est également reconnue par le jury extérieur, qui a classé ces équipes en bonne ou très bonne position $\left(1^{\mathrm{er}}\right.$ ou $\left.2^{\mathrm{e}}\right)$.

Dans la catégorie « équipe cohésive » (3 équipes), l'ensemble des membres puisent leur énergie de la bonne entente entre des personnes complémentaires et impliquées, facilitant le travail en commun. «La bonne humeur et la bonne entente entre tous les membres de l'équipe a été quelque chose de primordial dans la dynamique du groupe. Pour moi, il était important que tout le monde ait une attitude positive et que tout le monde soit soudé autour de ce projet pour qu'il avance au mieux et au plus vite ». «On a réussi à bien s'organiser et à ce que tout le monde joue le jeu, ce qui est très rare dans les autres cours. [...] Ce que je retiens de l'expérience, c'est vraiment l'importance de la cohésion d'équipe, de la complémentarité des compétences. » «Durant ce projet, j'ai eu l'occasion de travailler avec des personnes très agréables et sérieuses, ce qui a largement facilité tout le travail à effectuer et nous a permis d'éviter un certain nombre de contraintes. » La conflictualité apparaît nettement plus réduite que dans les autres catégories d'équipes (6 verbatim sur 87). Elle est même jugée insuffisante dans deux équipes sur trois : «Le projet a coulé un peu comme un long fleuve tranquille. Un peu de discorde, selon moi, aurait permis d'aboutir à quelque chose de plus original. La première leçon que je tire donc de ce projet est qu'une bonne entente et des amitiés fortes au sein d'un groupe ne sont pas forcément synonymes d'un projet parfait, loin de là. Il est peutêtre préférable de mettre, dans une moindre mesure, des personnalités aussi susceptibles de rentrer en contradiction et n'ayant pas peur du conflit. » «Le point fort de notre projet était vraiment la complémentarité des membres de l'équipe et l'entente générale. Atout principal selon moi mais aussi inconvénient majeur car l'absence de confrontations, de conflits internes, de contradictions a été facteur de consensus mou dans l'équipe. » Il existe peu de mentions d'une reconnaissance du leadership dans ces groupes (4 verbatim sur 48). Les fonctions de leadership les plus exercées concernent l'expert-pédagogue, l'ambassadeur ou le social comme le montrent les verbatim suivants provenant de deux équipes différentes : «Elle prenait beaucoup de notes quand nous discutions de notre projet, ce qui nous a permis de cadrer de plus en plus nos conversations, de les "orienter" et de nous faire tous travailler dans le même 
sens », «Le leader du groupe s'est naturellement présenté sous la personne de M. Il ne s'agissait pas ici de donner des directives mais réellement de motiver et de réunir tout le monde. Ainsi personne ne s'est senti mis à l'écart et la contribution de tous les membres se faisait naturellement tout au long des séances. » L'implication est contagieuse dans ces groupes : «J'ai été rapidement impressionnée par la qualité du travail fourni par plusieurs personnes, et cela m'a énormément motivée. Pour "faire aussi bien qu'elles", je me suis totalement prise au jeu et j'ai pris beaucoup de plaisir à travailler sur mes parties. » La qualité est perçue par les membres du groupe comme conforme aux attentes mais les jurys extérieurs ont émis des avis contrastés sur ces équipes : 2 équipes ont impressionné le jury (classées en $1^{\text {re }}$ ou $2^{\mathrm{e}}$ position) mais la $3^{\mathrm{e}}$ équipe a été classée avant-dernière.

Deux équipes sont caractérisées par un leader unique de type organisateur. Dans les deux cas, ce leader ne s'est mis en place que tardivement pour sauver le groupe séparé jusque-là en deux sous-groupes multiculturels ou avec des différences d'implication très fortes (passagers clandestins). «Dès le début nous avions repéré des difficultés de cohésion au sein du groupe, divisé très clairement en deux: un sous-groupe français et l'autre composé d'étudiants italiens ». L'ambiance est globalement mauvaise avec un nombre élevé de conflits (16 verbatim sur 87) majoritairement destructeurs ou non exprimés (12 verbatim sur 16). «L'écart trop flagrant entre le niveau d'implication des 2 sous-groupes a commencé à faire naître des tensions, les échanges étaient différents et la patience de chacun était mise à rude épreuve. » «Quelque chose s'est quand même brisé à ce moment-là : l'espoir de bonne entente entre les membres du groupe. Une limite avait été franchie. » «Nous ressentons J., S. et moi-même un véritable manque d'implication de la part des trois autres membres de l'équipe. Nous décidons d'attendre encore avant de leur en faire part. » Pour sauver le projet, un leader se met en place afin de déléguer les tâches et diriger le travail des passagers clandestins. Ce leader est très clairement nommé (30 verbatim sur 48) comme le montrent les verbatim suivants provenant de deux équipes différentes. « $G .{ }^{8}$ a fait preuve d'une force de management impressionnante. Elle connaissait bien les trois autres et arrivait à les mettre au travail en leur donnant des ordres clairs, simples et concis avec autorité. » «J. ${ }^{9}$ effectuait les mises à jour sur Trello, nous faisait des petits rappels sur Facebook et n'hésitait pas à remonter les bretelles à ceux qui ne

\footnotetext{
${ }^{8}$ G. appartient à une équipe de l'école européenne.

${ }^{9} \mathrm{~J}$. appartient à une équipe de l'école française.
} 
s'impliquaient pas assez. » Au final, la production est conforme aux attentes des membres du groupe $^{10}$ et jugée moyenne par les jurys extérieurs.

Dans les équipes à leadership en échec (5 équipes sur 13), il n’y a quasiment pas de reconnaissance spontanée de leader(s) par les membres des groupes (2 verbatim sur 48) et les fonctions de leadership exercées sont essentiellement de nature organisationnelle ${ }^{11}$. Mais on perçoit dans le récit une souffrance de cet organisateur, qui manque de reconnaissance de la part des autres membres. Ce qui semble signaler un leadership en échec. «J'avais l'impression de passer pour le "flic de service". » «S. me disait notamment qu'il était dans les temps et que ça ne servait à rien de le harceler. » «J'étais forcée de vérifier l'avancement de chaque mission afin de contrôler le travail effectué qui était souvent à retravailler et d'assigner de nouvelles missions sur le travail à faire. Ce rôle de project manager est assez fastidieux même pour quelqu'un qui aime l'organisation et qui est habitué à effectuer ce type de suivi. » "J'avais l'impression qu'on m'imposait la tâche la plus laborieuse [d'organisation], j'ai pris sur moi et j'ai accepté de faire cette partie pour le bien de l'équipe mais intérieurement, j'avais l'impression d'être seul contre tous. » La coopération est perçue comme faible, il y a des conflits récurrents (39 verbatim sur 87). La proportion de verbatim concernant les conflits destructeurs ou non exprimés (33 sur 39) est encore plus élevée que dans le cas des équipes à leader organisateur unique : «Un membre de l'équipe ne travaille pas, ne s'investit pas et se moque de l'avenir du groupe, les tensions se créent. Pour sauver le groupe, nous continuons de travailler sans nous soucier de lui, il sera mis de côté jusqu'à la fin du projet. Je ne pense pas être quelqu'un de dur ou cruel, mais ce qu'il pouvait penser ou dire pour justifier son inutilité, je m'en moquais, nous nous en moquions tous. » « Je pense que j'aurais dî m'énerver plus souvent et ne pas tout faire. » «Certains membres du groupe peuvent avoir du mal à reconnaître leurs talents et n'osent donc pas s'affirmer et prendre plus position dans le groupe. » «J'étais quelque peu frustrée de constater que je m'investissais peut-être plus que d'autres. Il n'était pas nécessaire à mes yeux, d'en parler plus longuement avec les autres. » Cela conduit à un désengagement progressif de tous. «C'était le pire moment du projet, quand je me suis rendu compte que trois membres du groupe n'étaient pas assez impliqués et ne coopéraient pas

\footnotetext{
${ }^{10}$ Les verbatim exprimant un résultat conforme aux attentes $(1,14$ et 1,83$)$ sont supérieurs aux verbatim exprimant un résultat inférieur aux attentes $(0,71$ et 0,17$)$. Il n'y a aucun verbatim exprimant un résultat supérieur aux attentes.

${ }^{11}$ La seule fonction de leadership mentionnée est de nature organisationnelle au sens d'une planification et d'un suivi des tâches. Mais toutes les équipes ne rattachent pas forcément l'organisation des tâches à la notion de leadership (verbatim compris entre 0 et 2). En revanche le thème de la division des tâches est exprimé dans toutes ces équipes et toujours associé à quelque chose qui s'est mal passé.
} 
du tout. » «Nous travaillions chacun de notre côté sans prévenir les autres de nos avancées. » «Chacun a son propre avis sur ses idées et il est impossible de parvenir à un compromis pour satisfaire tout le monde. » «Nous stagnions sur différentes étapes du projet, des tensions voire des brouilles entre collègues sont apparues. Chacun se plaignant du manque d'implication de l'autre. » Cette configuration aboutit à des résultats jugés majoritairement inférieurs aux attentes par les membres du groupe ${ }^{12}$ ainsi que par le jury. Les équipes concernées sont toutes classées en queue de classe. «Ce problème d'implication au sein du groupe fut bien sûr ressenti au niveau des résultats obtenus. »

\section{Discussion}

Nos résultats permettent d'affiner la connaissance sur les niveaux de coopération réels au sein d'équipe projets d'étudiants ainsi que leur effet sur la réussite perçue. Ceci constitue une contribution significative dans le champ de la pédagogie de l'entrepreneuriat au sujet de la dynamique des groupes. Ils viennent confirmer l'importance grandissante accordée dans des recherches récentes aux pratiques coopératives et collaboratives au sein des équipes d'étudiants en entrepreneuriat. Ils sont cohérents avec les recherches sur la résolution de conflits interpersonnels émergeant pendant les projets (Wang, Lai et Lu, 2020), ou l'exercice des mécanismes de confiance ou de contrôle influant sur la stabilité ou la fragilité des équipes entrepreneuriales dans l'atteinte de leurs objectifs (Williams Middleton \& Nowell, 2018). De plus, nos résultats peuvent appuyer de façon concrète l'appel de Witte (2014) d'inclure l'appropriation d'un esprit coopératif dans les curricula des programmes de management. De leur côté, Pepin, Tremblay et Audebrand (2019) suggèrent de repenser l'éducation entrepreneuriale afin d'intégrer la responsabilité individuelle et collective des futurs entrepreneurs et ainsi les armer pour mieux confronter les défis de notre société moderne. Nous pensons que l'apprentissage de la coopération à travers l'exercice conscient des fonctions de leadership peut y contribuer.

Les quatre types de groupe que nous avons identifiés nuancent les intuitions que nous avions au démarrage de la recherche. Comme nous le supposions, l'exercice effectif (ou non) des différentes fonctions de leadership a un impact réel sur le niveau de coopération perçu et sur la production résultante. Deux configurations positives se dessinent. Les équipes à leadership

\footnotetext{
12 Sur 4 des 5 équipes concernées, les verbatim exprimant un résultat perçu comme inférieur aux attentes ont des valeurs plus importantes $(0,8$ à 1,6$)$ que ceux exprimant un résultat conforme aux attentes $(0,6$ à 1,2$)$.
} 
distribué dans lesquelles sont présentes plusieurs fonctions de leadership, dont le social et l'organisateur (essentielles selon Barry, 1991) mettent en œuvre une coopération productive basée sur des valeurs de solidarité et de générosité aboutissant à une production supérieure aux attentes. Les équipes cohésives dans lesquelles il existe un leader social et un expert-pédagogue sans véritable leader organisateur, mettent en place une coopération orientée sur la générosité sans exagérer la pression sur la production, ce qui aboutit à des résultats conformes aux attentes. À l'inverse, les équipes caractérisées par un leadership en échec ne sont ni collaboratives ni coopératives. Les membres sont faiblement ou inégalement impliqués, ils se reconnaissent peu entre eux, la production est perçue comme inférieure aux attentes.

Notre recherche met en évidence un résultat inattendu dans le cas des équipes à leader organisateur unique. Ces équipes mettent en œuvre une collaboration au sens d'une coordination des tâches basée sur la solidarité et réussissent à produire. Mais la collaboration est vécue ici comme un pis-aller qui permet de sauver effectivement la production sans toutefois atteindre un résultat de bonne qualité aux yeux du jury comme des membres du groupe. En somme, l'équipe a fait ce qu'il faut pour sortir un résultat acceptable mais au prix d'une forte démotivation, dont témoignent certains verbatim : «J'aime que les gens s'entendent bien dans un groupe et qu'ils aiment travailler autour d'un projet commun. Ne pas arriver à cette situation m'a beaucoup troublée.... Je perdais de plus en plus ma motivation. 》 «L'écart entre les 2 groupes se faisait de plus en plus ressentir et je ne parvenais plus à répartir les tâches et à organiser le travail et le bon déroulement du projet comme je le faisais avant. Ma motivation commençait elle aussi à diminuer ce qui m'arrive très rarement dans ce type de projet. J'étais épris d'une grande lassitude vis-à-vis du projet et de l'école en règle générale. »

Ces résultats sont donc à double tranchant. Ils mettent en évidence les possibilités mais aussi les difficultés de la coopération dans les projets entrepreneuriaux étudiants.

Les deux premiers types de groupes (leadership distribué / équipe cohésive) explicitent les fonctions de leadership à exercer dans l'équipe afin de faire exister une perception de coopération parmi les membres. Ils mettent en évidence la complémentarité de trois fonctions principales de leadership (organisateur, social et expert-pédagogue) lesquelles sont exercées par des membres du groupe différents et peuvent être dosées différemment selon les équipes. Ces deux configurations de groupe permettent aussi de distinguer deux natures de coopération. Le premier type de groupe met en place une gestion consciente du leadership, progressivement 
partagée entre les membres. Il établit une coopération de production ${ }^{13}$ basée sur l'entraide et la valorisation des talents de chacun afin de relever l'ensemble des défis du projet qui émergent au fur et à mesure. La capacité du/des leaders naturel(s) au départ à verbaliser ses propres limites et à prendre conscience des besoins des autres membres apparaît comme une clé pour accéder à la distribution des fonctions. Le deuxième type de groupe plutôt porté par des valeurs de partage et d'harmonie entre les membres met au deuxième plan l'objectif de production et tend à éviter les conflits socio-cognitifs nécessaires pour aboutir à un projet ambitieux. Il s'agit alors plutôt d'une coopération d'entente basée sur le respect et l'amitié entre les membres. Coopérer suppose donc un choix de valeurs prioritaires (entraide ou harmonie, défi ou amitié) comme le souligne Go (2013). Nous ajoutons que cela suppose d'apprendre à exercer différentes fonctions de leadership lesquelles peuvent être conscientisées, discutées, apprises et gérées au sein de l'équipe. Si le groupe préfère l'harmonie et met en place spontanément un fonctionnement cohésif (correspondant à l'approche coopérative de Go (2013)), l'accompagnement du leadership ne semble pas nécessaire. En revanche, si le groupe tend à mettre en place un leadership organisateur unique, et que les conflits ne sont pas gérés, l'accompagnement par l'enseignant peut aider à basculer vers une modalité distribuée plus satisfaisante.

Notre recherche prend place dans un contexte de pédagogie entrepreneuriale dans l'enseignement supérieur qui présuppose un principe d'autodétermination s'appuyant sur l'autonomie des étudiants. Ce principe favorise plutôt une approche collaborative de la part de l'enseignant qui tend à laisser les groupes s'autogérer. Toutefois elle est à nuancer dans le cas des deux classes étudiées. En effet, les équipes ont bénéficié d'un accompagnement, au sens d'une explicitation des fonctions de leadership et d'un temps de coaching dédié à chacune. De plus, dans le cas de la classe française, plusieurs exercices spécifiques ont été proposés à différents moments du projet, notamment pour prendre conscience des talents de chacun. Un certain nombre de témoignages dans cette classe montrent que ces exercices ont eu un impact réel sur la compréhension de certains groupes quant au fonctionnement du leadership et sur la reconnaissance de chaque membre au sein du groupe : «Finalement, les différentes formes de leadership expliquées dans le cours d'accompagnement, nous ont permis de parler et à mes camarades de me demander de partager ma place. » « Je crois que les exercices dans le cadre des cours d'accompagnement m'ont beaucoup aidé. Notamment le jeu des enveloppes où nous

\footnotetext{
${ }^{13}$ Une étudiante explique que les attentes partagées au départ du projet par les membres de son équipe étaient de « en plus d'obtenir une bonne note, de créer une coopérative de salariés, avoir une certaine éthique pour l'entreprise et travailler sur un projet viable économiquement pour 5 entrepreneurs. »
} 
devions mettre tour à tour et personnellement les compétences de chacun et les axes d'améliorations à développer. Après cela, je me suis sentie plus reconnue dans mon groupe. » « Grâce à certains cours (dont ceux d'accompagnement de projet) j'ai réussi à mieux comprendre le fonctionnement de chacun et de m'adapter à eux. » Il n'en reste pas moins qu'une grande liberté d'organisation a été laissée aux groupes. Ce qui implique que les groupes peuvent échouer en termes de coopération et cela a un prix. C'est le cas des deux derniers types d'équipes.

Le troisième type d'équipe finit le projet envers et contre tout, grâce à l'émergence tardive d'un leader organisateur autoritaire. Celui-ci met au travail les passagers clandestins mais ne peut annuler l'existence des sous-groupes quelle que soit leur origine (genres, plusieurs cultures, hétérogénéité de niveaux), ce qui accentue les conflits, affaiblit la coopération perçue alors qu'elle est désirée et finalement génère de la souffrance. Ce constat rejoint le phénomène observé par Foliard et Le Pontois (2017) dans l'étude de leur cas unique via l'auto-confrontation croisée. L'équipe observée avait performé mais au prix d'une souffrance méconnue des accompagnateurs focalisés sur le succès du projet plutôt que sur les relations au sein du groupe. Les auteurs montrent que les étudiants se sont enfermés dans des identités de rôle (leader ou suiveur) qu'ils se sont eux-mêmes assignées inconsciemment par manque de verbalisation sur le leadership exercé au sein de l'équipe et sur la possibilité de le distribuer. En fin de projet, les liens d'amitié existants au départ se sont détériorés.

Dans notre recherche, le dernier type d'équipe fort répandu (5 équipes sur 13, soit 38 \%) ne réussit pas à mettre en place un leadership et souffre de toutes les difficultés : conflits non gérés, sous-implication, hétérogénéité entre les membres, manque de verbalisation des attentes et des rôles des uns et des autres. La coopération est impossible alors qu'elle est prescrite et désirée. La souffrance résultante est encore plus forte chez les étudiants qui tentent de prendre le leadership dans ces équipes mais échouent. En effet l'identité de rôle implicitement autoassignée comme leader est terriblement frustrée du fait d'une absence de reconnaissance ni par les résultats, ni par les membres du groupe. C'est ce que montrent les verbatim suivants : «Je suis épuisée, à bout. J'ai hâte de finir l'école. Je pense aussi avoir perdu mon amitié avec 2 membres de mon équipe à cause de leur manque de motivation et du fait qu'ils se reposaient sur moi tout le temps et que je n'ai eu aucune reconnaissance de leur part. » "J'ai eu du mal à entendre les arguments de mes camarades ("si c'est nous qui le faisons, on va avoir une mauvaise note, il ne faudra pas te plaindre", "tu es le plus douê" », "pense à l'intérêt du groupe" etc), J'ai pris sur moi et j'ai accepté de faire cette partie pour le bien de l'équipe, mais 
intérieurement j'avais l'impression d'être seule contre tous. Je pense que c'est surtout la manière de dire les choses qui m'a déplu. J'avais l'impression qu'on me faisait du chantage. » "Le pire moment du projet, c'est quand je me suis rendu compte que trois membres du groupe n'étaient pas assez impliqués et ne coopéraient pas du tout. Je me sentais mal. Soit, ils n'aimaient pas l'idée du projet, ou pire, ils n'étaient pas contents de m'avoir comme membre du groupe.»

Ces deux cas d'équipes qui ne réussissent pas à verbaliser un leadership inapproprié (excessivement autoritaire dans le premier cas, par défaut dans le deuxième) ont donc des conséquences négatives en termes de détérioration des liens d'amitié et de perte d'estime de soi. Ce risque met l'accent sur l'importance du rôle de l'enseignant pour soutenir les équipes afin d'expliciter les besoins de leadership et de leur permettre de découvrir les différentes possibilités de l'exercer.

\section{Conclusion}

Cette étude avait pour objectif d'affiner la connaissance des niveaux de coopération au sein des équipes projet d'étudiants en entrepreneuriat ainsi que leur influence sur la réussite des projets. Notre contribution met en évidence plusieurs niveaux de coopération, qui sont liés à la configuration de leadership mise en œuvre, laquelle influence effectivement la réussite telle qu'elle est perçue par les membres du groupe et par les jurys extérieurs.

Finalement, nous montrons que ni la réussite, ni la coopération ne sont acquises d'emblée. En réalité, 10 groupes sur 13, soit plus des 2/3, échouent au moins sur l'un des deux points. Seules les équipes à leadership distribué tiennent le double pari de produire un très haut niveau de qualité du projet et une satisfaction partagée par tous les membres, produisant une émulation coopérative contagieuse. Les autres équipes rentrent dans trois cas différents. Soit elles tiennent l'objectif coopératif aux dépens d'une production ambitieuse (3 équipes à groupe cohésif), soit elles réalisent l'objectif de production à un niveau acceptable mais au prix d'une souffrance dans les relations de groupe à moyen terme (2 équipes à leader organisateur), soit elles échouent sur les deux plans, ce qui peut générer une blessure identitaire pour ceux qui ont tenté d'être leaders sans obtenir de reconnaissance (5 équipes à leadership en échec).

Bien que notre étude porte sur un échantillon limité à 66 étudiants, nos résultats conduisent à nuancer l'idée reçue en pédagogie entrepreneuriale qu'il faut coopérer pour réussir. D'abord, la coopération n'explique pas tout ; lorsqu'elle est présente, la coopération peut effectivement 
conduire à une réussite exemplaire. Mais les équipes peuvent préférer la cohésion à la coopération, sacrifiant la qualité des résultats pour maintenir la bonne entente entre les membres. Elles peuvent aussi préférer la collaboration à la coopération, en organisant la division des tâches au risque de multiplier les conflits ou le désengagement de passagers clandestins. Mais d'autre part, le point essentiel est que la coopération ne va pas de soi. Coopérer suppose d'affronter les deux failles résumées par Baudrit (2007) l'improbable égalité d'implication lorsque les groupes sont autonomes dans leur gestion et l'impossible équité entre des personnes hétérogènes en termes de niveau, de culture ou de genre faisant naturellement émerger des leaders et des suiveurs. Dans notre contexte, les différentes fonctions de leadership apparaissent relativement partagées entre étudiants et étudiantes. Nous n'avons pas focalisé notre étude sur les différences de genre. Mais il se pourrait bien qu'elles aient un impact sur les attentes de rôle et sur la capacité des uns et des autres à prendre en charge et à se faire reconnaître en tant que leaders sur les différentes fonctions. De même, certains verbatim au sujet des conflits non résolus pourraient donner le sentiment qu'ils sont exacerbés dans les équipes multiculturelles. La comparaison du nombre de verbatim par rapport à la seule équipe française dans le cas de l'école européenne s'avère peu probante ${ }^{14}$. Ceci est peut-être dû à la taille de notre échantillon. Ce point mériterait des recherches approfondies sur des données plus larges.

Nos résultats questionnent également le cadre général de la pédagogie mise en place par l'enseignant dans la classe. Il ne suffit pas de laisser les apprenants s'autogérer pour qu'ils coopèrent, ni même pour qu'ils collaborent. Faire coopérer suppose donc d'entraîner les compétences sociales et métacognitives des apprenants afin qu'ils puissent prendre en charge tous les besoins des équipes, notamment à travers toutes les fonctions de leadership désirables au regard de leurs objectifs vis-à-vis du projet. Concrètement ${ }^{15}$, dès le démarrage du projet il est possible d'aider les étudiants à verbaliser leurs compétences relationnelles déjà existantes. L'un des exercices plébiscités dans la classe française s'appuie sur l'histoire scolaire et extrascolaire des étudiants afin de révéler des compétences existantes mais souvent ignorées par euxmêmes et par les autres membres du groupe. Un autre exercice s'appuie sur le partage d'expériences de projet antérieures avec la consigne de formuler un contrat ou une charte

\footnotetext{
${ }^{14}$ Une équipe française en leadership distribué, 2 équipes multiculturelles cohésives, 1 équipe multiculturelle à leader organisateur unique et 1 équipe multiculturelle à leadership en échec.

${ }^{15}$ Les exemples d'outils et méthodes proposés proviennent de l'expérience des auteurs. Il en existe beaucoup d'autres que l'on peut consulter dans le livre Raucent, B., Verzat, C., Villeneuve, L. (2010). Accompagner les étudiants, Bruxelles, De Boeck, nouvelle édition prévue en 2021.
} 
d'équipe qui pourra être rappelée dans les phases de tensions. En cours de projet, l'enseignant peut également aider l'équipe à découvrir et négocier les fonctions à distribuer. Le but sera toujours d'aider l'équipe à prendre conscience de ses choix et de leurs conséquences sur la progression du projet, sa réussite probable et sur les relations au sein du groupe. In fine, un travail réflexif a posteriori peut aider chaque étudiant.e à faire le point sur les compétences acquises et leurs conséquences en termes d'actions futures et de transformation identitaire. Le support utilisé comme matériau pour cette recherche abonde de commentaires à ce niveau et pourra faire l'objet d'une recherche ultérieure.

Ceci a des implications pratiques importantes en termes de posture pédagogique des enseignants. Il faut d'abord convaincre les enseignants de l'importance d'une double finalité éducative au sein des projets entrepreneuriaux, à savoir réussir le projet dans ses dimensions techniques, économiques, opérationnelles... mais également atteindre un haut niveau de coopération au sein des équipes d'étudiants. La deuxième implication pratique de cette recherche est de pousser les enseignants à adopter une posture d'accompagnement basée sur la facilitation plutôt que le guidage. La facilitation doit permettre aux étudiants d'exprimer leurs attentes vis-à-vis du leadership et à faciliter l'émergence de choix partagés. Elle s'appuie sur l'expression des émotions vécues au cours du projet. Ce n'est pas confortable pour des enseignants peu habitués à accompagner l'équipe sur le registre émotionnel.

Lors du débat Fishbowl ${ }^{16}$ qui a suivi cette présentation au sein du symposium sur l'accompagnement des équipes d'étudiants lors du colloque QPES 2019, deux thématiques clés sont ressorties : la question de la constitution des équipes d'étudiants et la capacité des enseignants quelle que soit leur origine disciplinaire à intégrer les dimensions relationnelles à composante émotionnelle dans leur accompagnement.

Les étudiants qui se sont choisis et/ou qui ont choisi leur projet feront-ils des groupes plus coopératifs ? Faut-il tenir compte des compétences techniques ou relationnelles déjà là ? Les témoignages semblent indiquer que toutes les formules sont intéressantes. Mais d'une part, la motivation semble supérieure lorsqu'il y a des possibilités de choix et lorsque l'on accepte que tous les étudiants n'aient pas les mêmes priorités d'apprentissage et d'ambition de réussite. D'autre part, la coopération semble supérieure et les équipes sont moins en difficulté lorsque l'enseignant aménage un espace spécifique pour que les étudiants puissent parler des choix

\footnotetext{
${ }^{16}$ Le débat Fishbowl (ou aquarium) est une méthode de facilitation des discussions en groupe dans laquelle les participants sont tour à tour acteur et auditeurs du débat. https://ressources.osons.cc/?BocalAPoissonsFishbawl
} 
opérés dans l'action et des émotions qui en résultent. La parole n'est pas obligatoire mais l'étudiant doit pouvoir s'exprimer s'il le souhaite. Des recherches ultérieures seraient à mener pour préciser ce qui constitue précisément cet espace de parole.

La deuxième question abordée concerne l'une des dimensions de ce cadre pédagogique : à savoir la posture de l'enseignant. Ses composantes essentielles ressorties du débat semblent être la bienveillance, le souci de générer des émotions positives grâce à des petits succès, mais aussi la modestie et la capacité à parler soi-même de ses émotions dans un groupe, produisant un effet de modelage sur les étudiants. Un consensus semble s'être opéré chez les participants sur le fait que les compétences de facilitation des relations et des émotions devraient faire partie du cœur du métier d'enseignant, alors que les enseignants sont généralement plus à l'aise sur le contenu de leur propre discipline. Dès lors, comment acquérir ces compétences de facilitation quand on n'est pas psychologue de formation? L'une des réponses proposées lors du débat Fishbowl est d'inviter les enseignants de différentes disciplines à vivre des travaux de groupe coopératifs et de disposer d'espaces réflexifs propices à confronter la variété de leurs perceptions. Les enseignants pourraient ainsi mieux comprendre les freins et leviers de la coopération du point de vue des étudiants mais aussi tester des outils, méthodes et postures d'accompagnement qui la favorisent. Plusieurs questions suivantes ont émergé : Comment motiver les collègues à participer à de telles formations expérientielles ? Cela transformerait-il les postures d'enseignants et par ricochet, les relations au sein des équipes d'étudiants? De nombreuses avenues de recherche se dessinent ici.

\section{Références bibliographiques}

Barry, D. (1991). Managing the bossless team: Lessons in Distributed Leadership. Organizational Dynamics, 1(20), 31-47.

Baudrit, A. (2007). Apprentissage coopératif/Apprentissage collaboratif : d'un comparatisme conventionnel à un comparatisme critique. Les Sciences de l'éducation - Pour l'Ère nouvelle, 1(40), 115-136.

Davidson, N. \& Major, C. H. (2014). Boundary Crossings: Cooperative Learning, Collaborative Learning, and Problem-Based Learning. Journal on Excellence In College Teaching, 25(3-4), $7-55$.

Foliard, S. \& Le Pontois, S. (2017). Équipes entrepreneuriales étudiantes : comprendre pour agir. Entreprendre \& Innover, 33(2), 44-54. https://doi.org/10.3917/entin.033.0044

Go, N. (2013). Approche coopérative et complexe en éducation. Dans M. Sumputh et F. Fourcade, Oser la pédagogie coopérative complexe (pp. 47-80). Lyon : Chronique Sociale. 
Johnson, D. \& Johnson, R. (1979). Conflict in the Classroom: Controversy and Learning. Review of Educational Research, 49(1), 51-79.

Pelletier, D. (2005). Invitation à la culture entrepreneuriale. Québec : Septembre éditeur. https://www.jeunes.gouv.qc.ca/publications/entrepreneuriat/invitation-culture.asp

Pepin, M., Tremblay, M. \& Audebrand, L. K. (2019). Towards a Conceptual Framework for Responsible Entrepreneurship: A Deweyan Perspective. Academy of Management Proceedings.

Peyrat-Malaterre, M. (2011). Comment faire travailler efficacement des élèves en groupe, tutorat et apprentissage coopératif. Bruxelles : De Boeck.

Raucent, B., Verzat, C. \& Villeneuve, L. (2010) Accompagner des étudiants - Quels rôles pour l'enseignant? Quels dispositifs? Quelles mises en cuvre? Bruxelles : De Boeck

Slavin, R. (1995). The Cooperative Learning : theory, research and practice. Boston: Allyn et Bacon.

Surlemont, B. \& Kearney, P. (2009). Pédagogie et esprit d'entreprendre. Bruxelles : De Boeck.

Verzat, C., O'Shea, N. \& Jore, M. (2017). Teaching proactivity in the entrepreneurial classroom. Entrepreneurship and Regional Development, 29(9-10), 975-1013.

Verzat, C., O'Shea, N. \& Raucent, B. (2015). Réguler le leadership dans les groupes d'étudiants en APP. Revue Internationale de Pédagogie de l'Enseignement Supérieur, 1(31), [En ligne].

Wang, T. U., Lai, W. Y. et Lu, C. T. (2020). Learning from others via team conflicts: Exploring the impact of individual entrepreneurial characteristics on the construction of entrepreneurial identity. International Journal of Entrepreneurship Behavior and Research, 26(2), 373-396.

Williams Middleton, K et Nowell, P. (2018). Team trust and control in new venture emergence. International Journal of Entrepreneurship Behavior and Research, 24(4), 882-910.

Witte A.E. (2014) Co-operation - the missing value of business education, Journal of Management Development, 33(4), 357-373. 